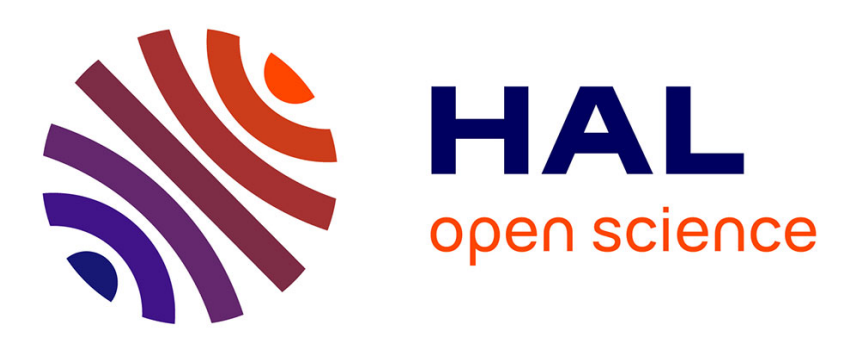

\title{
Approximation par la méthode des éléments finis de la formulation en domaine régulier de problèmes de fissures.
}

\author{
Zakaria Belhachmi, Jean-Marc Sac-Epee, Jan Sokolowski
}

\section{To cite this version:}

Zakaria Belhachmi, Jean-Marc Sac-Epee, Jan Sokolowski. Approximation par la méthode des éléments finis de la formulation en domaine régulier de problèmes de fissures.. Comptes Rendus. Mathématique, 2004, 338 (6), pp.499-504. 10.1016/j.crma.2004.01.008 . hal-02901533

\section{HAL Id: hal-02901533 \\ https://hal.science/hal-02901533}

Submitted on 17 Jul 2020

HAL is a multi-disciplinary open access archive for the deposit and dissemination of scientific research documents, whether they are published or not. The documents may come from teaching and research institutions in France or abroad, or from public or private research centers.
L'archive ouverte pluridisciplinaire HAL, est destinée au dépôt et à la diffusion de documents scientifiques de niveau recherche, publiés ou non, émanant des établissements d'enseignement et de recherche français ou étrangers, des laboratoires publics ou privés. 
Analyse numérique

\title{
Approximation par la méthode des éléments finis de la formulation en domaine régulier de problèmes de fissures
}

\author{
Zakaria Belhachmi ${ }^{\text {a }}$, Jean-Marc Sac-Epee ${ }^{\text {a }}$, Jan Sokolowski ${ }^{\text {b }}$

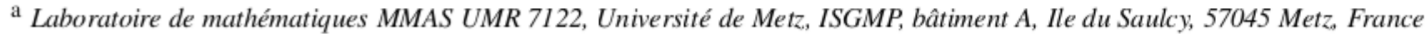 \\ b Institut Elie Cartan, laboratoire de mathématiques, Université Henri Poincare, Nancy I, BP 239, \\ 54506 Vandoeuvre-Les-Nancy cedex, France \\ Reçu le 25 septembre 2003 ; accepté après révision le 7 janvier 2004 \\ Présenté par Olivier Pironneau
}

\begin{abstract}
Résumé
Dans cette Note, nous présentons la discrétisation par différentes méthodes d'éléments finis mixtes d'une nouvelle formulation variationnelle d'un problème de fissure. Cette formulation variationnelle, dite méthode du domaine régulier, est ici mise en œuvre dans le cas d'un modèle simplifé de membrane élastique. Des conditions de type inégalité sont imposées sur les faces de la fissure, et le problème modèle se ramène à un problème de contact unilatéral sur cette fissure. L'analyse mathématique de ces méthodes d'éléments finis conduit à des taux de convergence optimaux énoncés ici. Pour citer cet article : Z. Belhachmi et al., C. R. Acad. Sci. Paris, Ser. I 338 (2004).

- 2004 Académie des sciences. Publié par Elsevier SAS. Tous droits réservés.
\end{abstract}

\section{Abstract}

Finite element approximation of the smooth domain formulation of crack problems. The discretization by various mixed finite element methods of a new variational formulation of crack problems is considered. The new formulation, called the smooth domain method, is derived for crack problems in the case of a simplified model of an elastic membrane. Inequality type boundary conditions are prescribed at the crack faces. The resulting model takes the form of an unilateral contact problem on the crack. The mathematical analysis for the method leads to optimal convergence rates, as given in this Note. To cite this article: Z. Belhachmi et al., C. R. Acad. Sci. Paris, Ser. I 338 (2004).

2004 Académie des sciences. Publié par Elsevier SAS. Tous droits réservés.

\section{Abridged English version}

\section{Introduction}

The numerical simulation of crack problems with unilateral contact conditions on the crack faces is source of many difficulties both in dealing with non-smooth domains and in the numerical modeling of contact conditions.

Adresses e-mail: belhach@math.univ-metz.fr (Z. Belhachmi), sokolows@iecn.u-nancy.fr (J. Sokolowski). 
For such problems, the possibility of performing the computations in the entire (smooth) domain without cracks reduce such difficulties and allows us to use the discretization by affine finite element methods in the 'more familiar' framework of classical variational inequalities arising from unilateral contact models $[4,2,3]$. This Note presents three mixed finite element methods for the approximation of the new variational formulation of unilateral crack model [5] analyzed in [1]. The optimal convergence rates are given in agreement with the numerical simulations.

\section{Problem formulation}

Let $\Omega$ be a bounded domain in $\mathbb{R}^{2}$ with smooth boundary $\Gamma$, and $\Gamma_{c} \subset \Omega$ be a smooth curve without selfintersections. We assume that $\Gamma_{c}$ can be extended to a closed smooth curve $\Sigma \subset \Omega$, with $\Sigma$ of class $C^{1,1}$, and $\Omega=\Omega^{1} \cup \Sigma \cup \Omega^{2}$ is divided into two sub-domains $\Omega^{1}, \Omega^{2}$ (see Fig. 1). In this case, $\Sigma=\partial \Omega^{1}$ is the boundary of $\Omega^{1}$ and $\Sigma \cup \Gamma=\partial \Omega^{2}$ is the boundary of $\Omega^{2}$. Let $\Omega_{c}$ be the domain $\Omega \backslash \overline{\Gamma_{c}}$, then $\Gamma_{c}$ is called a crack in the elastic body of the reference configuration $\Omega_{c}$. A simplified model of static equilibrium problem for an elastic membrane in the domain $\Omega_{c}$ with the interior crack $\Gamma_{c}$ is given below by (1) to (4). This problem admits a mixed variational formulation given by (5).

The smooth domain method is proposed in [5] for crack modelling and allows us to solve numerically the crack problem in the smooth domain $\Omega$. An application of the method means that the functions $\mathbf{p}$ and $u$ are extended to the entire domain $\Omega=\Omega_{c} \cup \Gamma_{c}$, and results in the closed variational formulation obtained by replacing $\Omega_{c}$ with $\Omega$ in (5). The well-posedness of smooth domain formulation is derived in [5] by a regularization technique.

\section{Discrete variational formulation}

We denote by $\mathcal{T}_{h}$ a family of regular triangulations of $\Omega$ made of triangular or quadrangular elements. Relying on the mixed variational formulation (11), where the inequality constraint on $\Gamma_{c}$ is expressed by a Lagrange multiplier, the approximation of solutions of the smooth domain formulation is performed by various mixed finite elements. We use Taylor-Hood element for the definition of the discrete finite element spaces associated to $u$ and $\mathbf{p}=\operatorname{grad} u$. The construction of the discrete spaces for the Lagrange multiplier is made with constant, respectively affine, piecewise functions defined on the 1D triangulation of $\Gamma_{c}$. The well-posedness of the resulting discrete problems is stated in Theorem 4.2. The convergence analysis uses a saddle point approach for the mixed formulations arising in unilateral contact problems. The final convergence rate is given in Theorem 4.3. Numerical simulations are also performed and are in agreement with the theoretical estimates [1].

\section{Introduction}

Cette Note présente une approximation de la nouvelle formulation variationnelle d'un modèle de fissure unilatéral [5] analysé dans [1], et donne des taux de convergence optimaux conformes aux simulations numériques effectuées. Le problème modèle (voir Fig. 1) que nous considérons peut se formuler comme suit : trouver $u$ telle que

$$
\begin{aligned}
& -\Delta u=f \quad \text { dans } \Omega_{c}, \\
& u=0 \quad \text { sur } \Gamma, \\
& {[u] \geqslant 0, \quad\left[\frac{\partial u}{\partial n}\right]=0, \quad[u] \frac{\partial u}{\partial n}=0 \quad \text { sur } \Gamma_{c},} \\
& \frac{\partial u}{\partial n} \leqslant 0 \quad \operatorname{sur} \Gamma_{c}^{ \pm},
\end{aligned}
$$

où $f$ est une fonction donnée de $L^{2}\left(\Omega_{c}\right)$. Le saut de $u$ sur $\Gamma_{c}$ est noté par $[u]=u^{+}-u^{-}$, où $u^{ \pm}=\left.u\right|_{\Gamma_{c}^{ \pm}}$désignent les traces de $u$ sur $\Gamma_{c}^{ \pm}$. 


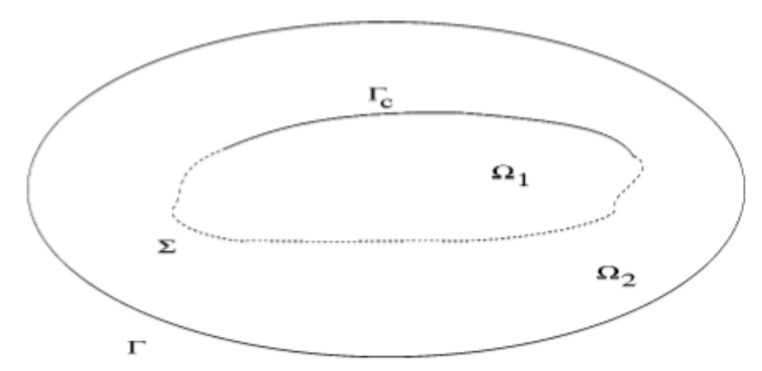

Fig. 1. Domaine avec fissure.

Fig. 1. Domain with a cut.

Nous notons $V=V\left(\Omega_{c}\right)$, l'espace $L^{2}\left(\Omega_{c}\right)$ et nous considérons l'espace $\mathbf{X}=\mathbf{X}\left(\Omega_{c}\right)$ :

$$
\mathbf{X}=\left\{\mathbf{q} \in L^{2}\left(\Omega_{c}\right)^{2}, \operatorname{div} \mathbf{q} \in L^{2}\left(\Omega_{c}\right)\right\} .
$$

Le problème (1)-(4) se formule à l'aide d'une inéquation variationnelle posée dans le cône convexe, fermé, $\mathbf{K} \subset \mathbf{X}$, que nous définissons grâce à l'espace de Sobolev $\left(H_{00}^{1 / 2}\left(\Gamma_{c}\right)\right)^{\prime}$ comme :

$$
\mathbf{K}=\left\{\mathbf{q} \in \mathbf{X},[\mathbf{q}, v]=0 \operatorname{sur} \Gamma_{c}(\mathbf{q} \cdot v)^{ \pm} \leqslant 0 \operatorname{sur} \Gamma_{c}^{ \pm}\right\},
$$

où [.] désigne le saut à travers $\Gamma_{c}$ et $v$ le vecteur unitaire extérieur normal à $\Omega^{1}$.

La formulation variationnelle mixte du problème aux limites (1)-(4) s'écrit alors :

Trouver $(\mathbf{p}, u) \in \mathbf{K}\left(\Omega_{c}\right) \times V\left(\Omega_{c}\right)$, tel que

$$
\left\{\begin{array}{l}
\int_{\Omega_{c}} \mathbf{p}(\mathbf{q}-\mathbf{p}) \mathrm{d} x+\int_{\Omega_{c}} u(\operatorname{div} \mathbf{q}-\operatorname{div} \mathbf{p}) \mathrm{d} x \geqslant 0, \quad \forall \mathbf{q} \in \mathbf{K}\left(\Omega_{c}\right), \\
-\int_{\Omega_{c}} \operatorname{div} \mathbf{p} v \mathrm{~d} x=\int_{\Omega_{c}} f v \mathrm{~d} x, \quad \forall v \in V\left(\Omega_{c}\right) .
\end{array}\right.
$$

\section{Formulation variationnelle dans le domaine régulier}

La nouvelle formulation du problème (5) consiste à remplacer le domaine fissuré $\Omega_{c}$ par $\Omega$ avec la modification qui en découle des espaces $\mathbf{X}=\mathbf{X}(\Omega)$ et $V=V(\Omega)$ est le nouveau convexe

$$
\mathbf{K}=\left\{\mathbf{q} \in \mathbf{X},(\mathbf{q} \cdot v) \leqslant 0 \text { sur } \Gamma_{c}\right\} .
$$

L'étude du problème, étendu à $\Omega$, est faite par régularisation. Pour $\delta>0$, on considère l'équation :

$$
\delta u_{\delta}-\operatorname{div} \mathbf{p}_{\delta}=f, \quad \text { dans } \Omega,
$$

elle mène au problème régularisé : trouver $\left(\mathbf{p}_{\delta}, u_{\delta}\right) \in \mathbf{K} \times V(\Omega)$ tel que

$$
\left\{\begin{array}{l}
a_{\delta}\left(u_{\delta}, v_{\delta}\right)+b\left(v_{\delta}, \mathbf{p}_{\delta}\right)=\left(f, v_{\delta}\right), \quad \forall v_{\delta} \in V=V(\Omega), \\
-b\left(u_{\delta}, \mathbf{q}_{\delta}-\mathbf{p}_{\delta}\right)+g\left(\mathbf{p}_{\delta}, \mathbf{q}_{\delta}-\mathbf{p}_{\delta}\right) \geqslant 0, \quad \forall \mathbf{q}_{\delta} \in \mathbf{K},
\end{array}\right.
$$

où

$$
a_{\delta}(u, v)=\delta \int_{\Omega} u v \mathrm{~d} x, \quad b(v, \mathbf{q})=-\int_{\Omega} v \operatorname{div} \mathbf{q} \mathrm{d} x, \quad g(\mathbf{p}, \mathbf{q})=\int_{\Omega} \mathbf{p q} \mathrm{d} x .
$$

La forme bilinéaire $a_{\delta}(\cdot, \cdot)$ est continue et $V$-elliptique, et $b(\cdot, \cdot)$ est continue et vérifie la condition inf-sup. Le résultat suivant est établi dans [5] :

Théorème 2.1. Le problème (7) admet une solution unique $\left(u_{\delta}, \mathbf{p}_{\delta}\right)$ pour toute fonction $f \in L^{2}(\Omega)$. De plus, on a l'estimation de stabilité suivante:

$$
\left\|u_{\delta}\right\|_{V}+\left\|\mathbf{p}_{\delta}\right\|_{X} \leqslant c\|f\|_{L^{2}(\Omega)},
$$

et la suite des solutions $\left(u_{\delta}, \mathbf{p}_{\delta}\right)_{\delta}$ converge vers la solution $(u, \mathbf{p})$ du problème $(5)$ quand $\delta$ tend vers 0. 


\section{Formulation variationnelle discrète}

Dans ce qui suit nous supposerons, pour simplifier l'exposé, que le domaine $\Omega$ est polygonal et que la fissure $\Gamma_{c}$ est un segment. On note $\mathcal{T}_{h}$ une famille régulière de triangulations de $\Omega$ par des triangles (ou des quadrilatères) de taille maximale $h$. Pour chaque élément $T$ de $\mathcal{T}_{h}, \mathcal{P}_{\ell}(T), \ell=0,1$, désigne l'espace des polynômes de degré $\ell$ sur $T$. Nous supposons que les extrémités de $\Gamma_{c}$ sont des sommets de $\mathcal{T}_{h}$. On désigne par $c_{1}=x_{0}, x_{1}, \ldots, x_{I-1}, x_{I}=c_{2}$ les nœuds sur $\Gamma_{c}$, et on pose $\left.t_{i}=\right] x_{i-1}, x_{i}$ [. Pour $h>0$, on introduit ensuite l'espace de dimension finie :

$$
V_{h}=\left\{v_{h} \in C(\bar{\Omega}), v_{h \mid T} \in \mathcal{P}_{1}(T)\right\} .
$$

Pour chaque élément $T$ de $\mathcal{T}_{h}$, la fonction bulle associée $\varphi_{T}$ est définie par :

$$
\varphi_{T}(x)=\prod_{i=1}^{3} \lambda_{i}(x) \quad \forall x \in T,
$$

où $\lambda_{i}$ désigne la $i^{\mathrm{e}}$ coordonnée barycentrique dans $T$. On définit $\mathcal{P}_{B}(T)=\mathcal{P}_{1}(T) \oplus \mathbb{R} \varphi_{T}$ comme espace d'approximation locale pour les champs de vecteurs dans $\mathbf{X}$. En conséquence, l'espace d'approximation globale s'écrit :

$$
\mathbf{X}_{h}=\left\{\mathbf{q}_{h} \in C(\bar{\Omega})^{2}, \mathbf{q}_{h} \in \mathcal{P}_{B}(T)^{2}\right\},
$$

et on introduit pour l'approximation de $\mathbf{K}$ le convexe fermé :

$$
\mathbf{K}_{h}=\left\{\mathbf{q}_{h} \in \mathbf{X}_{h}, \mathbf{q}_{h} \cdot v \leqslant 0 \text { sur } \Gamma_{c}\right\} .
$$

On note $V_{h}\left(\Omega^{\ell}\right)$ et $\mathbf{X}_{h}\left(\Omega^{\ell}\right), \ell=1,2$, les espaces de dimension finie de fonctions restreintes aux sous-domaines $\Omega^{\ell}$. Pour simplifier les notations, nous omettrons désormais le paramètre de régularisation $\delta>0$, et nous écrirons donc $u_{h}$ au lieu de $u_{\delta, h}$.

Le problème discret est défini de la manière suivante : trouver $\left(u_{h}, \mathbf{p}_{h}\right) \in V_{h} \times \mathbf{K}_{h}$ tel que

$$
\left\{\begin{array}{l}
a_{\delta}\left(u_{h}, v_{h}\right)+b\left(v_{h}, \mathbf{p}_{h}\right)=\left(f, v_{h}\right), \quad \forall v_{h} \in V_{h}, \\
-b\left(u_{h}, \mathbf{q}_{h}-\mathbf{p}_{h}\right)+g\left(\mathbf{p}_{h}, \mathbf{q}_{h}-\mathbf{p}_{h}\right) \geqslant 0, \quad \forall \mathbf{q}_{h} \in \mathbf{K}_{h} .
\end{array}\right.
$$

Le résultat suivant est démontré dans [1].

Théorème 3.1. Le problème (9) admet une solution unique $\left(u_{h}, \mathbf{p}_{h}\right)$ pour $h>0$. On a de plus l'estimation de stabilité suivante :

$$
\left\|u_{h}\right\|_{V}+\left\|\mathbf{p}_{h}\right\|_{X} \leqslant c\|f\|_{L^{2}(\Omega)}
$$

\section{Analyse de la convergence}

Comme nous avons choisi de prendre en compte la contrainte sur $\Gamma_{c}$ par un multiplicateur de Lagrange, nous commençons par introduire une nouvelle formulation mixte du problème (7). L'analyse mathématique de la nouvelle formulation repose sur la théorie du point selle et constitue une extension naturelle de celle rencontrée dans l'analyse de formulations variationnelles mixtes de problèmes de contact unilatéral sans frottement $[4,3,6,2]$. Nous considérons $\mathbf{X}_{h}$ muni de la norme, notée aussi $\|\cdot\|_{\mathbf{X}}$ :

$$
\left\|\mathbf{q}_{h}\right\| \mathbf{x}=\left(\sum_{T \in \mathcal{T}_{h}}\left\|\mathbf{q}_{h}\right\|_{L^{2}(T)^{2}}^{2}+h_{T}^{2}\left\|\operatorname{div} \mathbf{q}_{h}\right\|_{L^{2}(T)}^{2}\right)^{1 / 2} .
$$

On définit le cône convexe fermé :

$$
M=\left\{\mu \in H^{1 / 2}\left(\Gamma_{c}\right) ; \mu \geqslant 0\right\} .
$$


Le problème (7) admet alors la formulation équivalente :

Trouver $(u, \mathbf{p}, \lambda) \in V \times \mathbf{X} \times M$ tel que

$$
\left\{\begin{array}{l}
a_{\delta}(u, v)+b(v, \mathbf{p})=(f, v), \quad \forall v \in V, \\
-b(u, \mathbf{q})+g(\mathbf{p}, \mathbf{q})+\langle\lambda, \mathbf{q} \cdot v\rangle_{1 / 2, \Gamma_{c}}=0, \\
\langle\mu-\lambda, \mathbf{p} \cdot v\rangle_{1 / 2, \Gamma_{c}} \leqslant 0, \quad \forall \mu \in M,
\end{array} \quad \forall \mathbf{q} \in \mathbf{X},\right.
$$

où les inconnues $(u, \mathbf{p}, \lambda)$ sont le point selle du lagrangien associé à (11), défini sur l'ensemble produit $V \times \mathbf{X} \times M$.

Proposition 4.1. Le problème (11) admet une solution unique $(u, \mathbf{p}, \lambda) \in V \times \mathbf{X} \times M$ pour tout $\delta>0$. De plus,

$$
\lambda=[u] \operatorname{sur} \Gamma_{c} .
$$

Pour définir une méthode d'approximation du problème (11), nous introduisons deux espaces de dimensions finies sur $\Gamma_{c}$ :

$$
\begin{aligned}
& W_{h}^{0}\left(\Gamma_{c}\right)=\left\{\mu_{h}, \mu_{h \mid t_{i}} \in \mathbb{P}_{0}\left(t_{i}\right), 0 \leqslant i \leqslant I-1\right\}, \\
& W_{h}^{1}\left(\Gamma_{c}\right)=\left\{\mu_{h} \in C\left(\bar{\Gamma}_{c}\right), \exists \mathbf{q}_{h} \in \mathbf{X}_{h}, \text { tel que } \mathbf{q}_{h} \cdot v=\mu_{h} \text { sur } \Gamma_{c}\right\} .
\end{aligned}
$$

Nous pouvons alors, en fonction du choix d'un des deux espaces ci-dessus, définir plusieurs cônes convexes discrets $M_{h}$ pour l'approximation de $M$. Ainsi, le premier ensemble $M_{h}^{0}$ est défini par :

$$
M_{h}^{0}=\left\{\mu_{h} \in W_{h}^{0}\left(\Gamma_{c}\right), \mu_{h} \geqslant 0, \text { sur } \Gamma_{c}\right\} .
$$

Le choix de l'espace $W_{h}^{1}\left(\Gamma_{c}\right)$ conduit à l'un des ensembles,

$$
\begin{aligned}
& M_{h}^{1}=\left\{\mu_{h} \in W_{h}^{1}\left(\Gamma_{c}\right), \mu_{h} \geqslant 0, \operatorname{sur} \Gamma_{c}\right\}, \\
& M_{h}^{1, *}=\left\{\mu_{h} \in W_{h}^{1}\left(\Gamma_{c}\right), \int_{\Gamma_{c}} \mu_{h} \psi_{h} \mathrm{~d} \Gamma \geqslant 0, \forall \psi_{h} \in M_{h}^{1}\right\} .
\end{aligned}
$$

Remarque 1. Le choix $M_{h}^{0}$ est, à première vue, le moins favorable puisqu'il n'est pas un sous-espace de $M$. L'analyse de convergence confirme cette observation.

La solution $\left(u_{h}, \mathbf{p}_{h}, \lambda_{h}\right) \in V_{h} \times \mathbf{X}_{h} \times M_{h}$ de l'approximation mixte de dimension finie de (11) vérifie le système discret suivant d'équations et d'inégalités :

$$
\left\{\begin{array}{l}
a_{\delta}\left(u_{h}, v_{h}\right)+b\left(v_{h}, \mathbf{p}_{h}\right)=\left(f, v_{h}\right), \quad \forall v_{h} \in V_{h}, \\
-b\left(u_{h}, \mathbf{q}_{h}\right)+g\left(\mathbf{p}_{h}, \mathbf{q}_{h}\right)+\int_{\Gamma_{c}}\left(\lambda_{h}\right)\left(\mathbf{q}_{h} \cdot v\right) \mathrm{d} \sigma=0, \quad \forall \mathbf{q}_{h} \in \mathbf{X}_{h}, \\
\int_{\Gamma_{c}}\left(\mu_{h}-\lambda_{h}\right)\left(\mathbf{p}_{h} \cdot v\right) \mathrm{d} \sigma \leqslant 0 \quad \forall \mu_{h} \in M_{h},
\end{array}\right.
$$

où $M_{h}$ est un ensemble spécifique de multiplicateurs défini par (12), (13) ou (14).

Observons que l'unique solution $\left(u_{h}, \mathbf{p}_{h}\right)$ du problème (9) est également l'unique solution de (15). Pour les différentes méthodes d'approximation nous avons le résultat suivant :

Théorème 4.2. Supposons que l'ensemble $M_{h}$ soit donné par $M_{h}=M_{h}^{0}, M_{h}=M_{h}^{1}$ ou $M_{h}=M_{h}^{1, *}$. Pour chacun de ces choix, le problème (15) admet une solution unique. 
L'analyse de la convergence est résumée dans le théorème suivant :

Théorème 4.3. Soit $(u, \mathbf{p}, \lambda)$ la solution du problème (11). Supposons que $u_{\mid \Omega^{1}} \in H^{2}\left(\Omega^{1}\right), u_{\mid \Omega^{2}} \in H^{2}\left(\Omega^{2}\right)$ et de même que $\mathbf{p}_{\mid \Omega^{1}} \in H^{2}\left(\Omega^{1}\right)^{2}, \mathbf{p}_{\mid \Omega^{2}} \in H^{2}\left(\Omega^{2}\right)^{2}$. Soit $\left(\mathbf{U}_{h}, \lambda_{h}\right)$ la solution de (15) avec $M_{h}=M_{h}^{0}$. On a alors l'estimation :

$$
\left\|u-u_{h}\right\|_{V}+\left\|\mathbf{p}-\mathbf{p}_{h}\right\|_{\mathbf{x}}+\left\|\lambda-\lambda_{h}\right\|_{L^{2}\left(\Gamma_{c}\right)} \leqslant C(\delta, u, \mathbf{p}) h^{1 / 2} .
$$

Lorsque $M_{h}=M_{h}^{1, *}$, on a de même l'estimation :

$$
\left\|u-u_{h}\right\|_{V}+\left\|\mathbf{p}-\mathbf{p}_{h}\right\|_{\mathbf{x}}+\left\|\lambda-\lambda_{h}\right\|_{H^{1 / 2}\left(\Gamma_{c}\right)} \leqslant C(\delta, u, \mathbf{p}) h^{3 / 4},
$$

où $C(\delta, u, \mathbf{p})$ dépend linéairement de $\left\|u_{\mid \Omega^{\ell}}\right\|_{H^{2}\left(\Omega^{\ell}\right)}$ et $\left\|\mathbf{p}_{\mid \Omega^{\ell}}\right\|_{H^{2}\left(\Omega^{\ell}\right)^{2}}, \ell=1,2$.

Remarque 2. (i) Notons que l'erreur d'approximation ne dépend que de la régularité locale de la solution (i.e. de part et d'autre de la fissure).

(ii) Dans le cas $M_{h}=M_{h}^{1}$, l'ordre de convergence est en $\mathrm{O}\left(h^{1 / 2}\right)$, [2].

\section{Références}

[1] Z. Belhachmi, J.M. Sac-Epee, J. Sokolowski, Mixed finite element methods for smooth domain formulation of crack problems, soumis.

[2] F. Ben Belgacem, Y. Renard, Hybrid finite element methods for the Signorini problem, Math. Comp. 72 (243) (2003) $1117-1147$.

[3] P. Coorevits, P. Hild, K. Lhalouani, T. Sassi, Mixed finite element methods for unilateral problems: convergence analysis and numerical studies, Math. Comp. 71 (237) (2001) 1-25.

[4] J. Haslinger, I. Hlaváček, Contact between elastic bodies. 2. Finite element analysis, Apl. Mat. 26 (1981) $263-290$.

[5] A.M. Khludnev, J. Sokolowski, Smooth domain method for crack problems, Quart. Appl. Math., in press.

[6] K. Lhalouani, T. Sassi, Nonconforming mixed variational formulation and domain decomposition for unilateral problems, East-West J. Numer. Math. 7 (1999) 23-30. 